\title{
Preliminary hysteroscopic tubal hydrotubation improves fertility outcomes after laparoscopic salpingotomy for tubal ampullary pregnancy
}

Qing $\mathrm{Wu}^{1 \dagger}$, Yanling Lin ${ }^{2 \dagger}$, Jing Zhang ${ }^{1}$, Yun Zhou ${ }^{2}$, Lifeng Chen ${ }^{1}$ and Tan Lin ${ }^{2 *}$

\begin{abstract}
Background: Salpingotomy may change the anatomical structure and patency of the fallopian tube, which may affect the fallopian function. This study is to investigate the clinical efficacy of preliminary hysteroscopic tubal hydrotubation (HTH) after laparoscopic salpingotomy for tubal ampullary pregnancy.

Methods: A retrospective study was carried out, 140 women underwent laparoscopic salpingotomy for ampullary pregnancy from March 2013 to March 2017. Some patients received HTH in the 1st month and hysterosalpingography (HSG) in the 3rd month after salpingotomy (HTH group; $n=95$ ), and some patients only received HSG in the 3rd month after salpingotomy (control group; $n=45$ ). Clinical data, tubal patency and fertility outcome were evaluated after follow-up of 15 months.

Results: The tubal patency rate of the operated side was significantly higher in the HTH group than that in the control group (89.47\% vs 68.89\%, $P<0.05)$. The intrauterine pregnancy (IUP) rate was significantly higher in the HTH group (76.47\% vs $51.11 \%, P<0.05)$, and the recurrent ectopic pregnancy rate in the operated side was significantly lower in the HTH group than in the control group $(9.41 \%$ vs $22.22 \%, P<0.05)$. Logistic regression analysis showed that the positive factor for IUP was HTH $(\mathrm{OR}=3.109,95 \% \mathrm{Cl} 1.439-6.714, P=0.004)$, while the negative factors were history of pelvic inflammatory disease $(P I D)(O R=0.167,95 \% \mathrm{Cl} 0.074-0.377, P<0.001)$ and history of tubal infertility $(O R=0.286$, 95\% Cl 0.113-0.723, $P<0.05)$.

Conclusion: Preliminary HTH after laparoscopic salpingotomy for ampullary pregnancy could improve reproductive function and lead to a better fertility outcome. Patients without history of PID or tubal infertility may be the most suitable ones for HTH after salpingotomy.
\end{abstract}

Keywords: Laparoscopy, Ampullary pregnancy, Tubal hydrotubation, Fertility

*Correspondence: fisllintan@sina.com

${ }^{\dagger}$ Qing Wu and Yanling Lin contributed equally to this work

${ }^{2}$ Department of Obstetrics and Gynecology, Fujian Provincial Hospital,

Clinical Medical School of Fujian Medical University, 134, East Road,

Fuzhou 350001, Fujian, People's Republic of China

Full list of author information is available at the end of the article

\section{Background}

Ectopic pregnancy (EP) occurs approximately 1-2\% of all pregnancies [1]. Approximately $98 \%$ of ectopic pregnancies occur in the fallopian tube, and the ampullary portion is the most common site of implantation [2]. EP is a fertility-related problem for the reproductive-aged women desiring a future pregnancy [3]. Currently, laparoscopic surgery is the preferred treatment [4]. There 
are two types of surgical procedures for tubal pregnancy: the radical approach (salpingectomy) and the conservative approach (typically salpingotomy). Salpingectomy has been the standard procedure to remove ectopic pregnancy until laparoscopic salpingotomy was first introduced in 1993 by Bruhat et al. [5]

Many patients who desire to have children in future consider conservative surgery as the optimal treatment. Unfortunately, patients with salpingotomy can be at high risk of recurrent ectopic pregnancy (REP) and secondary infertility later on [6]. What's more, there is an increased incidence of persistent ectopic pregnancy (PEP) [7] in these patients.

At present, rare progress has been made to overcome the disadvantages of salpingotomy and reduce corresponding complications. In this study, the hysteroscopic tubal hydrotubation $(\mathrm{HTH})$ was hypothesized to be a beneficial method for the recovery of fallopian function and anatomical structure after salpingotomy. In our hospital, all patients were suggested to received HTH in the 1st month and hysterosalpingography (HSG) in the 3rd month after salpingotomy, while some patients rejected HTH and only received HSG in the 3rd month after salpingotomy to evaluate the patency of fallopian. We retrospectively analyzed data on the tubal patency and the clinical reproductive outcomes of these two groups to investigate the clinical efficacy of preliminary HTH.

\section{Material and methods Study subjects}

140 tubal ampullary pregnancy patients who received laparoscopic salpingotomy in the Department of Obstetrics and Gynecology in Fujian provincial hospital from March 2013 to March 2017 were enrolled. The study was approved by the board of Fujian provincial hospital ethics committee (No. 201400032), and preoperative informed consent was obtained from all patients after providing explanations of the possible risks and complications.

The following conditions were used as inclusion criteria for laparoscopic salpingotomy and this study: a visible ectopic ampullary tubal mass of natural conception by transvaginal sonography; preoperative serum $\beta$-hCG less than $10,000 \mathrm{mIU} / \mathrm{ml}$, maximal diameter of ectopic mass less than $5 \mathrm{~cm}$, a desire to maintain optimal tubal patency for future fertility; a minimum age of 18 years; an appropriate medical status for laparoscopic salpingotomy; and consent to surgical treatment and follow-up. Patients with recurrent tubal pregnancy, gynecologic malignancy, nontubal infertility diseases, incomplete records or follow-up were excluded.

\section{Surgical procedures}

All operations were performed by the same group of surgeons. Patients were placed in the trendelenburg position. In all cases, a $10-\mathrm{mm}$ trocar was inserted in the umbilicus, and three 5-mm trocars were inserted in the lower abdomen. A dilute solution of vasopressin (3 U vasopressin in $10 \mathrm{ml}$ saline solution) was injected into the mesosalpinx around the trouble tube to reduce blood loss. Salpingotomy involved a linear incision performed with scissors on the most prominent part of the fallopian tube, where correlated to the EP location. The ectopic mass was removed by the combination use of hydrodissection and traction with atraumatic forceps. Aspiration and compression lateral to the incision site were applied to facilitate the removal of the products, if necessary. The fallopian tube was closed in a single layer by two or three interrupted stitches using 3-0 VICRYL (Ethicon, USA). In all patients, methotrexate (MTX) $(1 \mathrm{mg} / \mathrm{kg})$ was injected locally into the mesosalpinx near the EP site.

\section{Hysteroscopic tubal hydrotubation (HTH)}

Some patients received HTH in the 1st month after salpingotomy. Surgery was performed within 3-7 day of obvious menstruation, and sex was prohibited for the remaining menstrual cycle. The bladder was emptied before surgery, and those patients with acute pelvic inflammatory disease (PID), vaginitis, or other contraindications were excluded. The cervix was grasped with a tenaculum and dilated to accommodate the 7-mm hysteroscope. The scope in the uterine cavity was advanced under direct visualization. The artificial plastic catheter was inserted about $1 \mathrm{~mm}$ into the uterotubal junction under hysteroscopy. Hydrotubation was carried out with a solution containing normal saline $20-40 \mathrm{ml}+$ chymotrypsin $1500 \mathrm{U}+$ dexamethasone $5 \mathrm{mg}+$ methylthioninium chloride injection ( $2 \mathrm{ml}: 20 \mathrm{mg}$ ) $0.1 \mathrm{ml}$ under $20-\mathrm{kPa}$ pushing pressure monitored by ultrasound scanning. The solution dispersed into the pelvic cavity if the tubes were unobtrusive and could be recanalized by hydrotubation.

\section{Hysterosalpingography (HSG)}

All patients received HSG in the 3rd month after laparoscopic salpingotomy. Surgery was performed within 3-7 day of obvious menstruation. HSG was performed in a standard sterile technique. The patient was placed in a lithotomy position, and a vaginal speculum was inserted. After cleaning the external os with povidoneiodine solution, the cervical os was cannulated with a balloon catheter. A cervical tenaculum was not used. The balloon catheter was inflated within the endocervical canal or lower uterine cavity, and contrast injection was performed with nonionic contrast medium iohexol 
injections, $20 \mathrm{ml} / 6 \mathrm{~g} / \mathrm{branch}$ (Beijing Hokuriku Pharmaceutical Co., Ltd., Beijing, China). The radiologists remotely administered the bolus injection using an automatic injection of $30 \%$ iohexol contrast agent at a volume of approximately $15 \mathrm{ml}$, according to the injection pressure and the patient's response to the regulated injection rate. The entire process was observed under timely and accurate radiography. Images of early and maximal opacification of the uterine cavity, fallopian tubes, and peritoneal contrast spillage were obtained. Fifteen minutes of photographic pelvic diffusion was recorded.

Fallopian tubal patency was assessed by HSG. The tubal patency was classified into three diagnostic grades: (a) patent, if the dye was seen in the whole tube during the injection, then totally disappeared from the tube but appeared in the pelvis 15 min later; (b) passable, if the dye was seen from the cornu to fimbria ends during the injection and more than two-thirds of the dye disappeared from the tube but was seen in the pelvis 15 min later; and (c) completely blocked, if the dye was not seen from the tubal cornu or no dye was seen in the pelvis 15 min later [8].

Patients were followed for up to 15 months after laparoscopic surgery. Data were analyzed to obtain a reproductive estimation. Intrauterine pregnancy (IUP) was verified by an ultrasound scans showing a fetal pole with a heartbeat. REP of the operated tube was diagnosed by laparoscopy and pathology, and secondary infertility was determined by performing interviews to assess if the patient failed to conceive for 1 year after HSG.

\section{Statistical analysis}

All statistical analyses were performed with SPSS 21.0 software (IBM, USA). Continuous variables were recorded as means \pm SDs. Categorical variables were described using proportions. Baseline patient characteristics were calculated via t-test for comparisons of normally distributed data and the rank-sum test for comparisons of non-normally distributed data. Count data were summarized as percentages and compared using the Chi-square test and Fisher's exact tests. Multivariate logistic regression was used to evaluate the relative factors. A P value $<0.05$ was considered statistically significant.

\section{Results}

All patients received HSG in the 3rd month after salpingotomy for future pregnancy. Based on whether patients received $\mathrm{HTH}$ in the 1st month after the operation, the patients were assigned into two groups: the HTH group $(\mathrm{n}=95)$ underwent HTH in the 1st month and HSG in the 3rd month after the operation, and the control group $(n=45)$ received only HSG in the 3rd month after salpingotomy.

The demographic clinical characteristics of the patients are displayed in Table 1 . The mean ages were $28.34 \pm 3.56$ years and $27.98 \pm 3.72$ years in both groups, respectively $(P=0.875)$. There were no significant differences in gravidity, parity, history of PID or history of tubal infertility between the two groups $(P>0.05)$. The mean preoperative serum $\beta$-hCG levels of the two groups were $3488 \pm 633.82 \mathrm{mIU} / \mathrm{ml}$ and $3415 \pm 617.88 \mathrm{mIU} / \mathrm{ml}$, and the mean durations of gestation were $43.82 \pm 2.77$ days and $43.44 \pm 2.78$ days $(P>0.05)$. The mean operative time and volume of bleeding were not significantly different. All ectopic pregnancies were confirmed by pathology. The $\beta$-hCG levels of all the patients in each group returned to baseline in less than 4 weeks after surgery. There were no procedural complications, such as reproductive systemic infection.

The patency rates of the operated tube after operation were $89.47 \%$ in the HTH group and $68.89 \%$ in the control group respectively, and the difference between the two groups was statistically significant $\left(\chi^{2}=9.109 ; P=0.003\right)$

Table 1 Baseline characteristics $(n=140)$

\begin{tabular}{llll}
\hline Characteristics & HTH group $(\mathbf{n}=\mathbf{9 5})$ & Control group $(\mathbf{n}=\mathbf{4 5})$ & $\boldsymbol{P}$ \\
\hline Age (years) & $28.3 \pm 3.6$ & $28.0 \pm 3.7$ & 0.875 \\
Gravidity (n) & 34 & 18 & 0.630 \\
Parity (n) & 16 & 6 & 0.594 \\
History of PID & 22 & 9 & 0.123 \\
History of tubal infertility (n) & 14 & $3415.0 \pm 617.9$ \\
Preoperative serum $\beta-h C G(\mathrm{mlU} / \mathrm{ml})$ & $3488.0 \pm 633.8$ & $43.4 \pm 2.8$ & 0.433 \\
Duration of gestation (days) & $43.8 \pm 2.8$ & $3.0 \pm 0.4$ & 0.564 \\
Maximal diameter of ectopic mass (cm) & $3.1 \pm 0.5$ & $49.4 \pm 7.7$ & 0.992 \\
Operative time (min) & $49.7 \pm 7.7$ & $17.9 \pm 5.8$ & 0.237 \\
Volume of bleeding (ml) & $16.8 \pm 4.9$ & & 0.157 \\
\hline
\end{tabular}


Table 2 The operated tubal patency rate of the two groups 3 months after the operation

\begin{tabular}{lllll}
\hline & $\mathbf{n}$ & Patent (\%) & Passable (\%) & $\begin{array}{l}\text { Completely } \\
\text { blocked (\%) }\end{array}$ \\
\hline HTH group & 95 & $85(89.47)$ & $7(7.37)$ & $3(3.16)$ \\
Control group & 45 & $31(68.89)$ & $8(17.78)$ & $6(13.33)$ \\
$P$ & & 0.003 & 0.063 & 0.022 \\
\hline
\end{tabular}

(Table 2). The patency rates of the contralateral side tube were $88.42 \%$ in the HTH group and $82.22 \%$ in the control group, and the difference between the two groups was not significant $(P=0.317))$.

Ten patients in the HTH group dropped out to conceive after HSG. In the 15th month after surgery, the IUP rates were $76.47 \%$ in the $\mathrm{HTH}$ group and $51.11 \%$ in the control group $\left(X^{2}=8.652 ; P=0.003\right)$. The REP rates of the operated side were $9.41 \%$ in the HTH group and $22.22 \%$ in the control group $\left(\chi^{2}=4.048 ; P=0.04\right)$. There were significant differences $(P<0.05)$ in the IUP rate and REP rate between the two groups. The secondary infertility rates were $14.11 \%$ and $26.67 \%$ in the HTH and control groups, respectively, without a significant difference $\left(x^{2}=3.078\right.$; $P=0.08$ ) (Table 3).

Multivariable analysis analyzed the influence factors for fertility outcome was listed in Table 4. Logistic regression analysis showed that the positive factor for IUP was $\mathrm{HTH}$ (odds ratio $[\mathrm{OR}]=3.109,95 \%$ confidence interval [CI] 1.439-6.714 $P=0.004)$, while the negative factors were history of PID (OR $=0.167,95 \%$ CI $0.074-0.377$ $P<0.001)$ and history of tubal infertility $(\mathrm{OR}=0.28695 \%$ CI 0.113-0.723 $P<0.05)$.

\section{Discussion}

EP is a severe gynecological problem among reproductive-aged women. In recent years, laparoscopy has become preferable to laparotomy for the treatment of tubal EP [9]. In clinical practice, there are two types of surgical procedures for tubal pregnancy: salpingectomy and salpingotomy. The choice of salpingotomy versus salpingectomy depends on many factors, including the age of the patient, the tube condition, the serum human

Table 3 IUP rate, REP rate and secondary infertility rate during the 15 months after the surgery

\begin{tabular}{lllll}
\hline & $\mathbf{n}$ & IUP rate (\%) & REP rate (\%) & $\begin{array}{l}\text { Secondary } \\
\text { infertility rate } \\
\mathbf{( \% )}\end{array}$ \\
\hline HTH group & 85 & $65(76.47)$ & $8(9.41)$ & $12(14.11)$ \\
Control group & 45 & $23(51.11)$ & $10(22.22)$ & $12(26.67)$ \\
$P$ & & 0.003 & 0.044 & 0.079 \\
\hline
\end{tabular}

Table 4 Logistic regression analysis discussing relative factors for fertility outcome

\begin{tabular}{llll}
\hline & OR & $\mathbf{9 5 \%} \mathrm{Cl}$ & $\boldsymbol{P}$ \\
\hline HTH & 3.109 & $1.439-6.714$ & 0.004 \\
History of abortion & 0.725 & $0.306-1.714$ & 0.464 \\
History of PID & 0.167 & $0.074-0.377$ & 0.000 \\
History of tubal infertility & 0.286 & $0.113-0.723$ & 0.008 \\
\hline
\end{tabular}

chorionic gonadotropin levels, the diameter of the tubal mass and the patient's future fertility desire.

The advantages and disadvantages of salpingotomy for EP have been debated for many years [10,11]. Although ectopic pregnancy lesions are conservatively cleared by salpingotomy, the change of the anatomical structure and patency of the fallopian tube may affect the fallopian function, resulting in decreased fecundity [12].

Salpingotomy also has several disadvantages. One disadvantage of salpingotomy is that it may increase the risk of PEP. A large tubal mass in EP is a high-risk factor for PEP after laparoscopic salpingotomy; therefore, $\beta$-hCG monitoring is mandatory [13]. Several studies reported that the rate of persistent trophoblastic disease was reduced by the use of prophylactic MTX in laparoscopic salpingotomy, which could be administered by local MTX injection into the mesosalpinx near the site of the EP $[12,14]$. In this study, all patients were injected with local MTX, and no patients suffered from PEP after laparoscopic salpingotomy.

Women with previous EP have higher incidence of REP and miscarriage in their second pregnancies [13]. Infertility and EP commonly occur due to causes such as tubal obstruction and pelvic adhesion [15]. Some studies compared subsequent fertility after salpingectomy to that after salpingotomy and found no statistically significant differences in the fertility outcomes [16, 17]. Turan et al. reported no significant differences in IUP rates up to 24 months after surgical treatment in younger patients undergoing salpingectomy compared to those undergoing salpingotomy [18]. However, several studies reported conflicting findings that there were higher risks for REP or lower cumulative IUP rates after laparoscopic salpingotomy $[9,19,20]$. Bennetot et al. reported that the 24-month cumulative rate of IUP was $76 \%$ after medical treatment, $67 \%$ after conservative surgery, and $67 \%$ after radical salpingostomy. The corresponding REP rates were $25.5 \%, 18.5 \%$, and $18.5 \%$ within 2 years, respectively [21]. In this study, the REP rate in the HTH group was $9.41 \%$, which was even lower than that reported result, and the 12-month cumulative rate of IUP is $76.47 \%$ in the $\mathrm{HTH}$ groups. In this study, logistic regression analysis showed that the positive factor for IUP was $\mathrm{HTH}$, while the 
negative factors were history of PID and history of tubal infertility.

As the structure and function of the fallopian tube need to be rebuilt after laparoscopic salpingotomy, it would be great to have an effective approach which can improve the recovery of fallopian and pelvic function. Recently, hysteroscopic techniques are highly accurate and sensitive for detecting fallopian tubal obstruction [22]. Therefore, the HTH was hypothesized as a beneficial approach for the recovery of fallopian. To our knowledge, this is the first study on the efficacy of HTH after salpingotomy with the aim of improving the patency of the fallopian tube and reducing complications.

In this study, HTH was performed 3-7 day after the first postsurgical menses in 95 patients. This procedure provided an opportunity not only to check the tubal patency and uterine cavity but also to assist fluid going through the inner cavity of the fallopian tube with hydrotubation. We hypothesized that tubal hydrotubation after salpingotomy could reduce blood coagulation and inflammatory factors, which may prompt the recovery of fallopian function and anatomical structure. Besides, the drugs used in hydrotubation are anti-inflammatory and may promote the recovery of oviduct. Lei et al. reported that hysteroscopic hydrotubation solution consisted of hydrocortisone $(20 \mathrm{mg})$, gentamicin $(160,000 \mathrm{IU})$ and procaine $(80 \mathrm{mg})$ in $20 \mathrm{ml}$ distilled water and treated the tubal blockage [23]. Our study also shows that patients who have undergone HTH have better potential fallopian and fertility outcomes. However, this study still has some shortcomings. Firstly, it is a retrospective cohort study with a limited sample size. Secondly, it is necessary to organize a prospective randomized controlled trial with multiple centers to confirm the results. As all surgical procedures were performed by the same surgery group in this study, the generalizability of the results may be weakened. Moreover, the mechanism of fallopian recovery should be further studied by animal models and clinical trial, especially, the mechanism of promoting effect of hysteroscopic tubal hydrotubation should be investigated.

\section{Conclusion}

This study indicated that the tube-preserving surgery by salpingotomy followed by HTH could represent an option for ectopic tubal pregnancy in women with a strong desire for fertility. Besides, based on the results of logistic regression analysis in this study, patients without history of PID or tubal infertility might be the most suitable ones for HTH after salpingotomy, which could achieve the most satisfactory fertility outcome.

\section{Abbreviations}

HTH: Hysteroscopic tubal hydrotubation; EP: Ectopic pregnancy; REP: Recurrent ectopic pregnancy; PEP: Persistent ectopic pregnancy; HSG: Hysterosalpingography; MTX: Methotrexate; PID: Pelvic inflammatory disease; IUP: Intrauterine pregnancy.

\section{Acknowledgements}

We are grateful to everyone involved in carrying out the study, analysing the data, and producing the manuscript.

\section{Authors' contributions}

QW and TL conceived the study and participated in its design, drafting and writing the manuscript as well as supervising the study and critically revising the manuscript. YL, YZ and QW collected the clinical data and research experiment. QW, LC and JZ were responsible for drafting and writing the manuscript and statistical analysis. All authors read and approved the final manuscript.

\section{Funding}

This study was supported by Zhejiang health science and technology program project (2021 KY504). The funders had no role in the study design, data collection and analysis, decision to publish, or preparation of the manuscript.

\section{Availability of data and materials}

The data of study are not publicly available due to ethical and legal restrictions. However, upon request, data may be available from the corresponding author on reasonable request.

\section{Declarations}

\section{Ethics approval and consent to participate}

Ethics Committee approval was obtained from the Institutional Ethics Committee of Fujian provincial hospital for the commencement of the study (No. 201400032). Informed consent was obtained from all patients.

\section{Consent for publication}

Not applicable.

\section{Competing interests}

The authors have declared that no competing interests exist.

\section{Author details}

${ }^{1}$ Department of Gynecology, Reproductive Medicine Center, Zhejiang Provincial People's Hospital, Affiliated People's Hospital, Hangzhou Medical College, Hangzhou 310014, Zhejiang, People's Republic of China. ${ }^{2}$ Department of Obstetrics and Gynecology, Fujian Provincial Hospital, Clinical Medical School of Fujian Medical University, 134, East Road, Fuzhou 350001, Fujian, People's Republic of China.

Received: 7 February 2021 Accepted: 10 October 2021

Published online: 12 February 2022

\section{References}

1. Kirk E, Bottomley C, Bourne T. Diagnosing ectopic pregnancy and current concepts in the management of pregnancy of unknown location. Hum Reprod Update. 2013;20(October 2):250-61.

2. Bouyer J, Coste J, Fernandez H, Pouly JL, Job-Spira N. Sites of ectopic pregnancy: a 10 year population-based study of 1800 cases. Hum Reprod. 2002;17(12):3224-30.

3. Hajenius PJ, Mol F, Mol BW, et al. Interventions for tubal ectopic pregnancy. Cochrane Database Syst Rev. 2007;24:CD000324.

4. Newbatt E, Beckles Z, Ullman R, et al. Ectopic pregnancy and miscarriage: summary of NICE guidance. BMJ. 2012;345:e8136.

5. Bruhat MA, Pouly JL. Endoscopic treatment of ectopic pregnancies. Curr Opin Obstet Gynecol. 1993;5(2):260-6.

6. American College of Obstetricians and Gynecologists. ACOG Practice Bulletin no 94: medical management of ectopic pregnancy. Obstet Gynecol. 2008;111:1476-84. 
7. Ting WH, Lin $\mathrm{HH}$, Hsiao SM. Factors predicting persistent ectopic pregnancy after laparoscopic salpingostomy or salpingotomy for tubal pregnancy: a retrospective cohort study. J Minim Invasive Gynecol. 2019;26(6):1036-43.

8. Ding $\mathrm{Y}$, Huang $\mathrm{W}$, Jiang $\mathrm{H}$, et al. A new tubal classification system for fertility prognosis after laparoscopic salpingostomy for tubal pregnancy. Eur J Obstet Gynecol. 2016;203:136-41.

9. Rana P, Kazmi I, Singh R, Afzal M, Al-Abbasi FA, Aseeri A, Singh R, Khan R, Anwar F. Ectopic pregnancy: a review. Arch Gynecol Obstet. 2013;288(4):747-57.

10. Clausen I. Conservative versus radical surgery for tubal pregnancy. A review. Acta Obstet Gynecol Scand. 1996;75(1):8-12.

11. Mol F, van Mello NM, Strandell A, et al. Salpingotomy versus salpingectomy in women with tubal pregnancy (European surgery in ectopic pregnancy study): an openlabel, multicenter, randomized controlled trial. Lancet. 2014;383:1483-9.

12 Fernandez H, Capmas P, Lucot JP, Resch B, Panel P, Bouyer J. Fertility after ectopic pregnancy: the DEMETER randomized trial. Hum Reprod. 2013;28:1247-53.

13. Madhra M, Horne AW. Ectopic pregnancy. Obstet Gynaecol Reprod Med. 2014;24(7):215-20.

14. Kaya H, Babar Y, Ozmen S, et al. Intratubal methotrexate for prevention of persistent ectopic pregnancy after salpingotomy. J Am Assoc Gynecol Laparosc. 2002;9(4):464-7.

15. Marion LL, Meeks GR. Ectopic pregnancy: history, incidence, epidemiology, and risk factors. Clin Obstet Gynecol. 2012;55:376-86.

16. Jamard A, Turck M, Pham AD, Dreyfus M, Benoist G. Fertility and risk of recurrence after surgical treatment of an ectopic pregnancy (EP): salpingostomy versus salpingectomy. J Gynecol Obstet Biol Reprod (Paris). 2016:45:129-38.

17. Kuroda K, Takeuchi H, Kitade M, et al. Assessment of tubal disorder as a risk factor for repeat ectopic pregnancy after laparoscopic surgery for tubal pregnancy. J Obstet Gynaecol Res. 2009;35:520-4.

18. Turan V. Fertility outcomes subsequent to treatment of tubal ectopic pregnancy in younger Turkish women. J Pediatr Adolesc Gynecol. 2011;24:251-5

19. Tulandi T, Guralnick M. Treatment of tubal ectopic pregnancy by salpingotomy with or without tubal suturing and salpingectomy. Fertil Steril. 1991;55:53-5.

20. Bangsgaard N, Lund CO, Ottesen B, et al. Improved fertility following conservative surgical treatment of ectopic pregnancy. BJOG. 2003;110:765-70.

21. de Bennetot M, Rabischong B, Aublet-Cuvelier B, et al. Fertility after tubal ectopic pre-gnancy: results of a population-based study. Fertil Steril. 2012;98:1271-6

22. Vitale SG, Carugno J, Riemma G, et al. Hysteroscopy for assessing fallopian tubal obstruction: a systematic review and diagnostic test accuracy metaanalysis. J Minim Invasive Gynecol. 2021;28(4):769-78.

23. Lei ZW, Xiao L, Xie L, Li J, Chen QX. Hysteroscopic hydrotubation for treatment of tubal blockage. Int J Gynaecol Obstet. 1991;34(1):61-4.

\section{Publisher's Note}

Springer Nature remains neutral with regard to jurisdictional claims in published maps and institutional affiliations.

Ready to submit your research? Choose BMC and benefit from:

- fast, convenient online submission

- thorough peer review by experienced researchers in your field

- rapid publication on acceptance

- support for research data, including large and complex data types

- gold Open Access which fosters wider collaboration and increased citations

- maximum visibility for your research: over 100M website views per year

At BMC, research is always in progress.

Learn more biomedcentral.com/submissions 